\title{
Review
}

Yong Chen*

\section{The structural biology of the shelterin complex}

https://doi.org/10.1515/hsz-2018-0368

Received September 10, 2018; accepted October 15, 2018; previously published online October 20, 2018

\begin{abstract}
The shelterin complex protects telomeric DNA and plays critical roles in maintaining chromosome stability. The structures and functions of the shelterin complex have been extensively explored in the past decades. This review summarizes the current progress on structural studies of shelterin complexes from different species. It focuses on the structural features and assembly of common structural domains, highlighting the evolutionary plasticity and conserved roles of shelterin proteins in telomere homeostasis and protection.
\end{abstract}

Keywords: DNA binding; protein-protein interaction; shelterin complex; structural biology; telomere; telomeric protein.

\section{Introduction}

Telomeres are specialized nucleoprotein complexes that cap the end of linear chromosomes to maintain genomic stability (de Lange, 2005; Palm and de Lange, 2008). Telomere DNA contains a long tract of duplex repetitive DNA region and a $3^{\prime}$ single-stranded (ss) G-overhang. A group of proteins specifically bind double-stranded (ds) and single-stranded telomeric DNAs to form a protective cap for shielding telomeres from inappropriate DNA repair (de Lange, 2005, 2009; Palm and de Lange, 2008). They also collaborate with telomerase to maintain telomere length (Smogorzewska and de Lange, 2004; Nandakumar and Cech, 2013; Martinez and Blasco, 2015). The removal and mutation of these telomeric proteins activate DNA damage response pathways and trigger the degradation or fusion

*Corresponding author: Yong Chen, State Key Laboratory of Molecular Biology, National Center for Protein Science Shanghai, Shanghai Science Research Center, CAS Center for Excellence in Molecular Cell Science, Shanghai Institute of Biochemistry and Cell Biology, Chinese Academy of Sciences, University of Chinese Academy of Sciences, 333 Haike Road, Shanghai 201210, China; and School of Life Science and Technology, Shanghai Tech University, 100 Haike Road, Shanghai 201210, China, e-mail: yongchen@sibcb.ac.cn. https://orcid.org/0000-0001-5857-6086 events of chromosomes (Palm and de Lange, 2008; de Lange, 2009).

These telomeric proteins usually form protein complexes to cooperatively regulate telomere homeostasis and stability (Palm and de Lange, 2008). Mammalian telomeres are bound by shelterin, a specialized six-protein complex that consists of TRF1, TRF2, RAP1, TIN2, TPP1 and POT1 proteins (Figure 1A and B) (de Lange, 2005). TRF1 and TRF2 exist as homodimers and bind the double-stranded DNA regions of telomeres with high affinity and specificity. TRF1 negatively regulates telomere length by a counting mechanism and promotes the efficient replication of telomere DNA (van Steensel and de Lange, 1997; Sfeir et al., 2009; Zimmermann et al., 2014). TRF2 primarily protects telomeres from being recognized as double-stranded DNA breaks (DSB) by inhibiting ataxia telangiectasia mutated (ATM) DNA-damage signaling pathway and non-homology end joining (NHEJ) repair pathway (Denchi and de Lange, 2007; de Lange, 2009). RAP1 is recruited to telomeres through interaction with TRF2. RAP1 functions together with TRF2 to inhibit homologous recombination (Martinez et al., 2010; Sfeir et al., 2010; Chen et al., 2011; Benarroch-Popivker et al., 2016; Rai et al., 2016). The 3' G-overhang is coated by ssDNA binding protein POT1 (Baumann and Cech, 2001). POT1 interacts with TPP1 to form a stable heterodimer. They function together to repress ataxia telangiectasia and Rad3-related (ATR) signaling pathway and regulate telomerase activity (Hockemeyer et al., 2006; Wu et al., 2006; Denchi and de Lange, 2007; Wang et al., 2007; Xin et al., 2007). TIN2 is a bridging molecule that simultaneously interacts with TRF1, TRF2 and TPP1 and facilitates the assembly of an intact shelterin complex (O'Connor et al., 2006; Hu et al., 2017). As the central hub of shelterin complex, TIN2 contributes to telomere length regulation and telomere protection (Kim et al., 1999; Takai et al., 2011; Frescas and de Lange, 2014; Hu et al., 2017).

Similarly, the telomeric complex with a shelterin-like architecture has also been identified in fission yeast Schizosaccharomyces pombe (Figure 1A and C). The S. pombe shelterin complex is composed of six proteins, namely, Taz1, Rap1, Poz1, Tpz1, Pot1 and Ccq1 (Miyoshi et al., 2008; Moser and Nakamura, 2009). Taz1, an ortholog of mammalian TRF1 and TRF2, directly binds double-stranded 
A

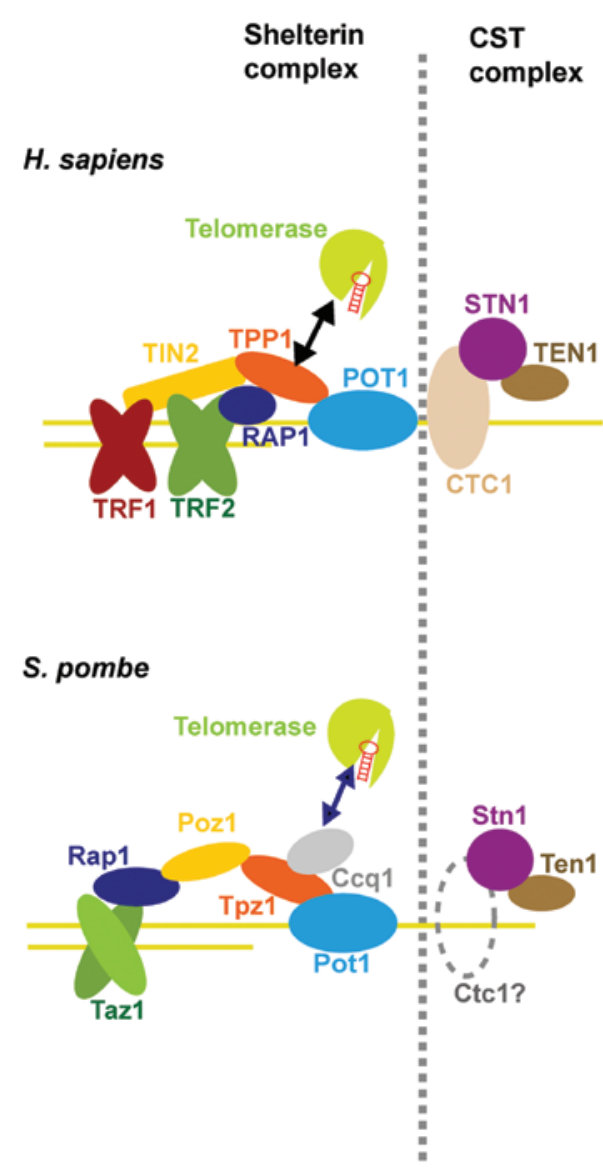

B H. sapiens Shelterin complex

ssDNA

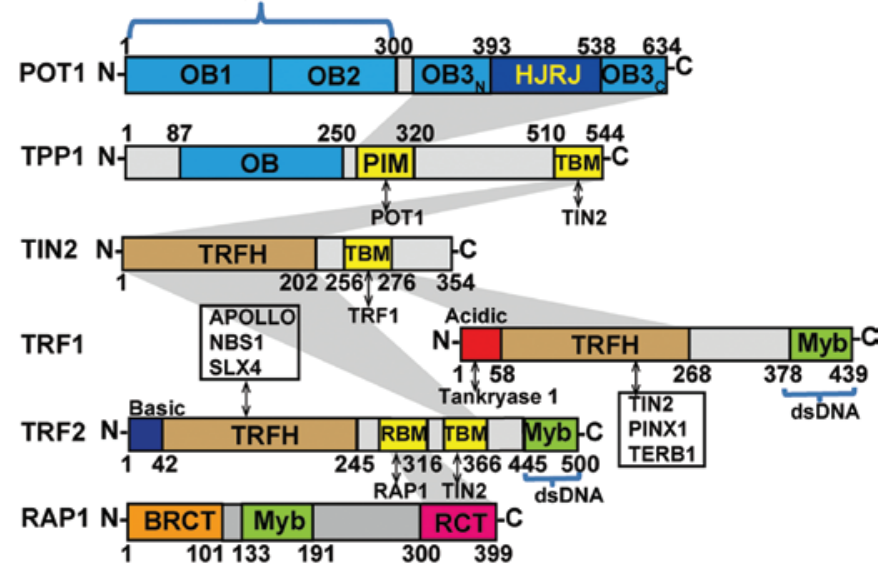

C S. pombe Shelterin complex
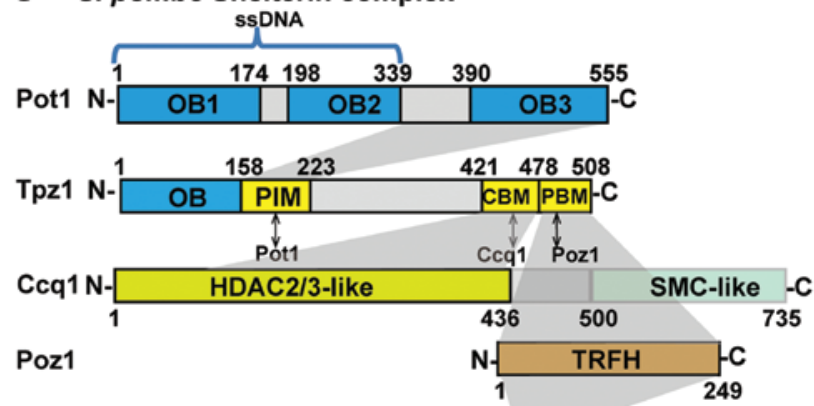

Rap1 N- BRCT $\mid$\begin{tabular}{|l|l|l|l|l|l|l|l|}
\hline Myb & & Myb & BIM & PBM & BBM & RCT & R \\
\hline
\end{tabular}

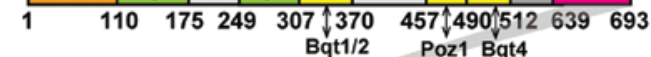

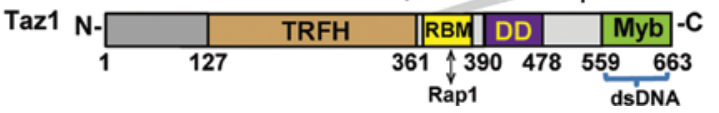

Figure 1: Compositions and interaction networks of shelterin complexes.

(A) Schematic diagram of the telomere-associated proteins in S. pombe and $H$. sapiens. (B) Domain organization of $H$. sapiens shelterin complex. Conserved domains and motifs are donated. Shaded areas indicate the identified bipartite interaction regions. (C) Domain organization of $S$. pombe shelterin complex.

telomeric repeats. Taz1 plays important roles in telomere synthesis, replication, recombination and protection (Cooper et al., 1997, 1998; Miller and Cooper, 2003; Miller et al., 2006). Taz1 recruits Rap1, an ortholog of mammalian RAP1, to telomeres (Kanoh and Ishikawa, 2001; Miller et al., 2005). Rap1 inhibits telomerase-dependent telomere elongation (Kanoh and Ishikawa, 2001; Miller et al., 2005). The single-stranded telomere-binding protein Pot1 forms a heterodimer with Tpz1. Pot1 and Tpzl are the orthologs of mammalian POT1 and TPP1, respectively (Miyoshi et al., 2008; Moser and Nakamura, 2009). The Pot1-Tpz1 heterodimer is critical to telomere stability, as removal of either pot1 or tpz1 leads to rapid telomere loss and chromosome circularization (Baumann and Cech, 2001; Miyoshi et al., 2008). Poz1, an ortholog of mammalian TIN2, interacts with Rap1 and Tpz1 simultaneously (Kim et al., 2017). Poz1 bridges the
ssDNA-binding subcomplex to dsDNA-binding subcomplex, and is important for telomere homeostasis (Miyoshi et al., 2008; Kim et al., 2017; Xue et al., 2017). Ccq1, a unique component in fission yeast, binds Tpz1 and mediates the recruitment of telomerase to telomeres (Tomita and Cooper, 2008).

Unlike mammalian and fission yeast cells, the budding yeast Saccharomyces cerevisiae does not have a shelterin-like complex. Rap1 is the major binder and organizer of telomeres in S. cerevisiae (Conrad et al., 1990; Azad and Tomar, 2016). It plays important roles in telomere length regulation, subtelomeric gene silencing and telomere end protection (Conrad et al., 1990; Kabir et al., 2010; Chen et al., 2011). Rap1 also serves as a docking platform for other telomere-associated proteins, including Sir-family proteins (Sir3 and Sir4) important to gene silencing, and Rif-family proteins (Rif1 and Rif2) crucial to 
telomere length regulation (Moretti and Shore, 2001; Shi et al., 2013). The 3' G-overhang region is bound by $\mathrm{Cdc13}$ proteins (Nugent et al., 1996), which associates with Stn1 and Ten1 to form a CST (Cdc13, Stn1, Ten1) complex. CST complex is a telomere-dedicated replication protein A (RPA) complex (Gelinas et al., 2009; Sun et al., 2009), and is essential to chromosome capping and telomere replication (Nugent et al., 1996; Churikov et al., 2013). Recent data show that CST complexes are also present in mammalian cells (Figure 1A), compete with POT1-TPP1 for telomere ssDNA binding, and regulate telomerase-mediated elongation and end protection (Miyake et al., 2009; Chen et al., 2012; Wu et al., 2012). The progress on structural studies of the CST complex has been extensively reviewed (Rice and Skordalakes, 2016). In this review, we focus on the shelterin complex to highlight the common structural features and evolutionary plasticity of shelterin proteins from different species.

\section{The building blocks in shelterin proteins}

Notably, shelterin proteins often contain some common structural domains connected by flexible loops (Lewis and Wuttke, 2012). These domains are the basic functional units of shelterin proteins. There are two DNA-binding modules: the oligonucleotide or oligosaccharide-binding (OB) fold and myeloblastosis (Myb) domain and three protein-binding modules: telomere repeat factor homolog (TRFH), RAP1 C-terminal (RCT) and BRCA1 C-terminal (BRCT) domains.

\section{OB fold: a single-stranded DNA-binding domain}

The OB fold is a common domain that is usually found in ssDNA- and RNA-binding proteins. OB folds are exclusively found in the 3' G-overhang associating shelterin components, including mammalian POT1-TPP1 and fission yeast Pot1-Tpz1 (Figure 1B). There are five OB folds in the human shelterin complex and four $\mathrm{OB}$ folds in the fission yeast shelterin complex. The available structures of shelterin $\mathrm{OB}$ folds have revealed the remarkable structural conservation and functional diversity of this fold (Lei et al., 2003, 2004; Wang et al., 2007; Nandakumar et al., 2010; Dickey et al., 2013; Chen et al., 2017; Rice et al., 2017). The OB fold is characterized by five $\beta$-strands that form a closed $\beta$-barrel structure (Figure $2 \mathrm{~A}$ ). The $\mathrm{N}$-terminal region and the connecting loop between $\beta 3$ and $\beta 4$ seal the top and bottom of the $\mathrm{OB}$ barrel, respectively. The shelterin-specific OB folds often end with a C-terminal $\alpha$-helix (Figure 2A). The loops connecting the $\beta$ strands are variable in length and usually contain extra structural elements that are important to functional specification. For example, the loop connecting $\beta 1$ and $\beta 2$ (L12) of $\mathrm{HsPOT}_{\mathrm{OB} 3}$ contains a 150-residues Holliday junction resolvase-like (HJRL) domain that is involved in TPP1-binding (Chen et al., 2017; Rice et al., 2017).

The primary function of $\mathrm{OB}$ fold is to recognize ssDNAs. The DNA-binding domains in HsPOT1 and SpPot1 are composed of tandem OB folds. Two OB folds in HsPOT1 form a compact unit to bind ssDNA, and the removal of any OB fold disrupts DNA-binding activity (Lei et al., 2004). By contrast, the two OB folds in fission yeast Pot1 work independently, and each OB fold retains its own DNA-binding activity and specificity (Croy et al., 2009). The different binding modes may be correlated to the distinct telomere sequences of these two species: the degenerate repeats in fission yeast and the well-defined repeats in human cells. Notwithstanding varied binding modes, each OB fold in HsPOT1 and SpPot1 recognizes ssDNA in a similar way. The ssDNA primarily binds in a groove formed by one side of the $\beta$-barrel and two flanking loops L12 and L45 (Figure 2B-D). The hydrogen bonding and aromatic stacking interactions between an OB fold and nucleotide bases assure specific telomeric sequence recognition (Lei et al., 2003, 2004; Nandakumar et al., 2010; Dickey et al., 2013).

Apart from DNA-binding functions, the OB fold is involved in protein-protein interactions. For example, POT1 uses its third OB fold to interact with a polypeptide from TPP1 (Chen et al., 2017; Rice et al., 2017). Interestingly, TPP1 is bound to $\mathrm{POT}_{\mathrm{OB}}$ in a similar groove used by ssDNA binding to OB1/2 of POT1 (Figure 2E), suggesting a conserved binding mode for OB folds in shelterin proteins. However, OB folds in other complexes (e.g. CST complex) use different binding interfaces in OB-fold-mediated interactions. Overall, an $\mathrm{OB}$ fold is a versatile interaction domain with common architecture and plays diversified functions in shelterin complexes.

\section{Myb domain: a double-stranded DNA-binding domain}

The Myb domain, or related homeodomain, is a classic double-stranded DNA binding domain with three $\alpha$-helices arranged in an orthogonal bundle. Myb domains are only identified in dsDNA-associating proteins, including TRF1, TRF2 and RAP1. Mammalian TRF1 
A
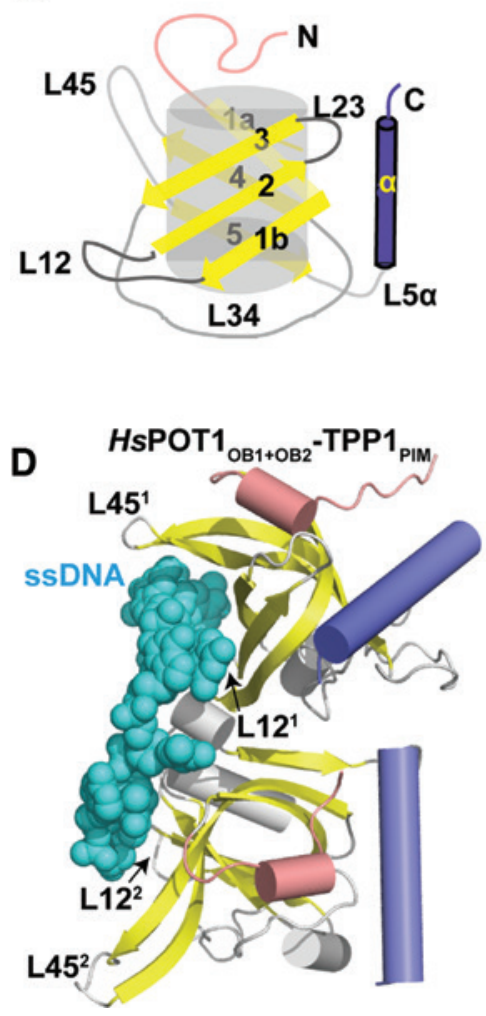

B
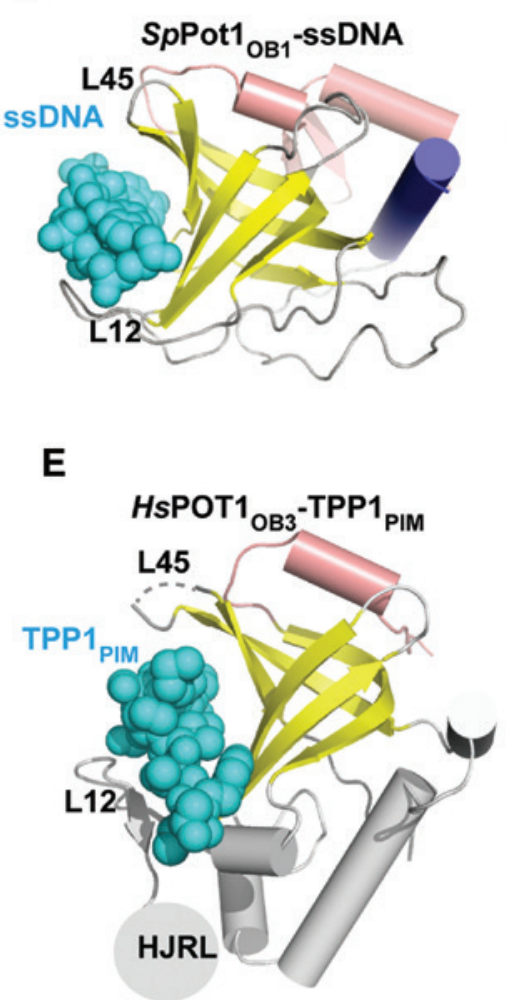

C

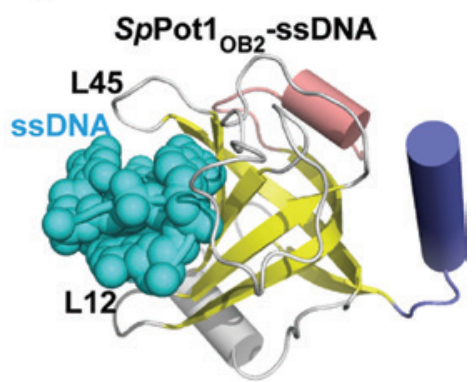

$\mathbf{F}$

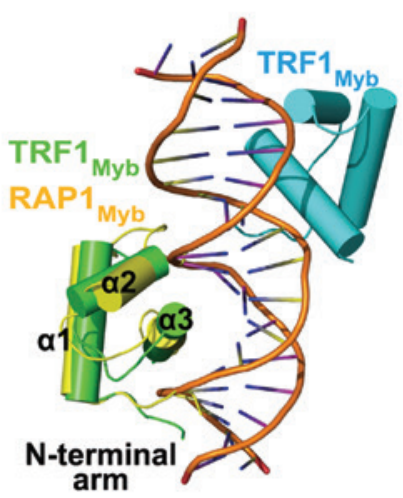

Figure 2: The DNA-binding domains in shelterin complexes.

(A) Topology of telomeric OB folds. The five $\beta$-stands (yellow) wrap around the central core. The connecting loops between $\beta$-stands are shown in gray. The $\mathrm{N}$-terminal and C-terminal extension are shown in salmon and marine, respectively. The same color codes are used in the following panels. (B) Structure of $S p P$ Pot $1_{\mathrm{OB}}{ }_{1}$-SSDNA complex (PDB: $\left.1 Q Z H\right)$ ). SpPot ${ }_{\mathrm{OB} 1}$ is in cartoon representation and ssDNA is shown as spheres in cyan. (C) Structure of $S p$ Pot $1_{\text {ов2 }}$-ssDNA (9mer) complex (PDB: 4HID). For clarity, the first six nucleotides are shown as spheres, and the last three nucleotides are omitted. (D) Structure of $H s P O T 1_{\text {ОВ1+OB2 }}$ in complex with 10mer SSDNA (PDB: $\left.1 X J V\right)$. (E) Structure of human POT1-TPP1 complex (PDB: 5H65). For clarity, the HJRL domain inserted in the POT1 ${ }_{\mathrm{OB} 3}$ and the portion of TPP1 interacting HJRL domain are omitted. HsPOT1 ${ }_{\mathrm{OB} 3}$ is in cartoon representation and TPP1 $1_{\mathrm{PIM}}$ (POT1-Interacting Motif) is shown as spheres in cyan. (F) Superimposition of HsRAP1 $1_{\text {Myb }}$ (PDB: 1FEX, yellow) with HsTRF1 ${ }_{\text {Myb }}$-dsDNA (PDB:1W0T). Two TRF1 ${ }_{\text {Myb }}$ domains (green and cyan) bind to a 19bp dsDNA.

and TRF2, and their fission ortholog Taz1 contain a single C-terminal Myb domain that specifically recognizes telomeric DNA. The crystal structures of Myb domains from TRF1 and TRF2 reveal the conserved binding mode that is present in all Myb-dsDNA complexes (Figure 2F) (Court et al., 2005). The $\alpha 2$ and $\alpha 3$ form a helix-turn-helix motif, which enables sequence-specific contacts with bases in the major groove of a DNA. The N-terminal arm interacts with the minor groove of a DNA to enhance affinity and specificity. Although the single Myb domain from TRF1/ TRF2/Taz1 can bind a telomeric DNA, these full-length proteins have 10-fold higher DNA-binding affinities than separated Myb domains (Bianchi et al., 1999; Deng et al., 2015). This phenomenon can be explained by the dimeric nature of TRF1/TRF2/Taz1 in vivo that enables two Myb domains in a functional unit to simultaneously recognize telomeric repeats with high affinities. The crystal structures of TRF1 and TRF2 Myb domains in complex with 19-bp telomeric dsDNA show that two Myb domains are bound to the opposite faces of two adjacent binding sites (Figure 2F) (Court et al., 2005). Notably, the long loop between dimerization domain and Myb domain provides a conformational flexibility to accommodate the varied spatial requirements in different conditions.

Rap1 proteins in fission yeast and mammalian cells also contain one or two Myb domains, but they do not have any DNA-binding activity. On the contrary, the budding yeast ortholog ScRap1 binds telomeric dsDNA directly via its tandem Myb domains. The nuclear magnetic resonance (NMR) structure of human RAP1 ${ }_{\text {Myb }}$ shows that RAP1 ${ }_{\text {Myb }}$ lacks significant positively charged residues on the surface in contrast to ScRap1 and thus unable to bind DNA (Hanaoka et al., 2001). Whether the Myb domains in RAP1 mediate protein-protein interactions and the functional roles of these Myb domains merit further investigation. 


\section{TRFH domain: a universal protein-interaction domain}

The TRFH domain was first identified in human TRF1 and TRF2 as a conserved domain sharing $27 \%$ sequence identity (Broccoli et al., 1997). The TRFH domains from human TRF1 and TRF2 have nearly identical architectures and adopt all-helix dimeric structures, as indicated by their crystal structures (Figure 3A) (Fairall et al., 2001). Each monomer contains $10 \alpha$-helices, and dimer formation is mediated by $\alpha 1, \alpha 2$ and $\alpha 10$ from each monomer (Figure 3A). The fission yeast ortholog of TRF1/2, Taz1, shows a similar monomeric structure but has a bent $\alpha 1$, and thus the dimerization interface is disrupted in Taz$1_{\text {TRFH }}$ (Deng et al., 2015). A short helical bundle between TRFH and Myb domain mediates the dimerization of
Taz1 (Deng et al., 2015). Recently, the crystal structures of human TIN2 and S. pombe Poz1 were determined (Hu et al., 2017; Kim et al., 2017; Xue et al., 2017). Unexpectedly, TIN2 and Poz1, despite their low sequence similarity, adopt TRFH-like folds with noticeable structural variation. The dimerization interface observed in TRF1 and TRF2 is not maintained in TIN2 $2_{\text {TRFH }}$, so that TIN2 $2_{\text {TRFH }}$ exists as a monomer. Meanwhile, Poz1 ${ }_{\mathrm{TRFH}}$ adopts a different dimeric conformation in an antiparallel arrangement mainly through $\alpha 1$ and $\alpha 2$ helices (Figure 3A).

TRFH domains are important protein-protein interaction domains that are essential for shelterin assembly and function. TRF $1_{\text {TRFH }}$ and TRF $2_{\text {TRFH }}$ serve as general docking platforms for various telomeric or telomere-associated proteins. The TRFH domains in TRF1 and TRF2 recognize a conserved motif, termed as the TRFH-binding-motif (TBM;
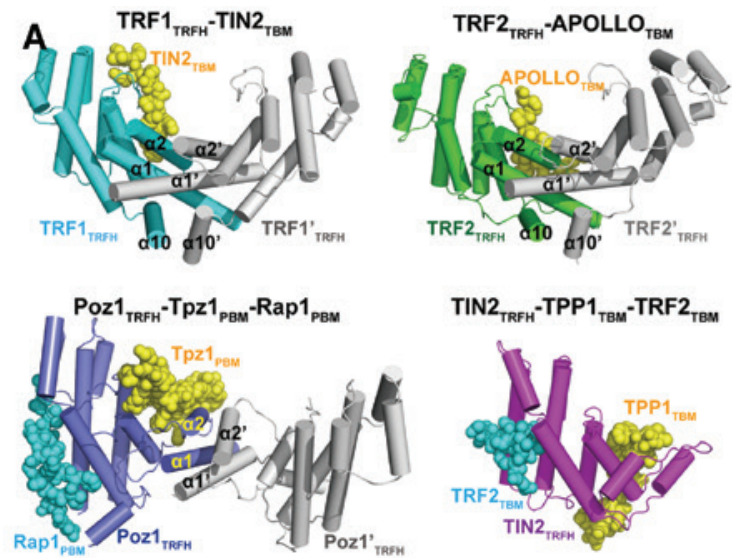

C

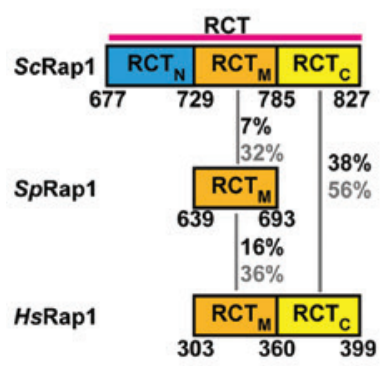

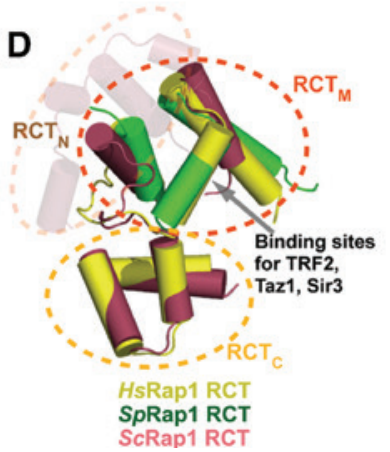

B

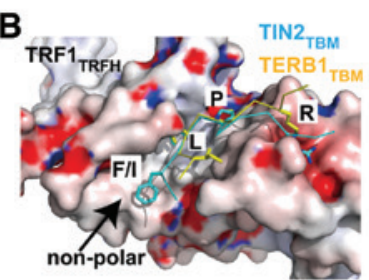
TRF1-binding motifs \begin{tabular}{ll}
\hline TIN2 & GRHFNIAPLGRRR \\
PINX1 & GRDFTLKPKKRRG
\end{tabular} TERB1 NKKILLTPRRROR Consesus: $\mathbf{x \times x F \times L \times P x ( K / R ) _ { 3 }}$

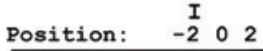
Telomere length regulation Telomere attachment to the NE

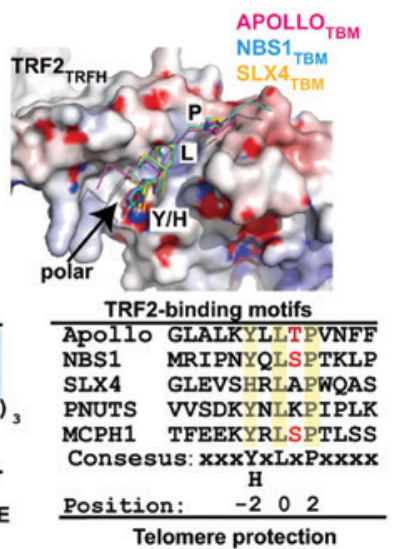

E

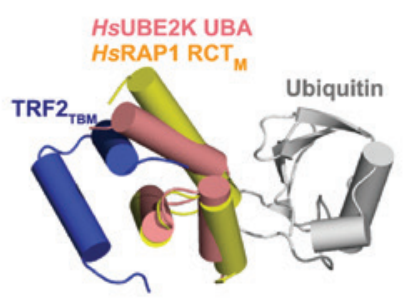

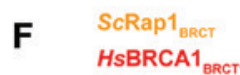

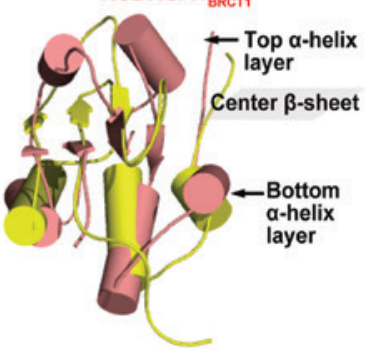

Figure 3: The protein-interaction domains in shelterin complexes.

(A) Crystal structures of TRF1-TIN2 (PDB: 3BQ0), TRF2-Apollo (PDB: 3BUA), Poz1-Tpz1-Rap1 (PDB: 5XXF) and TIN2-TPP1-TRF2 (5XYF) shown at the same orientation. The bound peptides are shown as spheres. The helices in the dimerization interfaces are indicated. (B) Detailed interaction interfaces and consensus binding motifs for TRFH domains from TRF1 and TRF2. Conserved residues important for TRFH-peptide interaction are shown in stick models in the structural model and shaded in sequence alignments. Potential phosphorylation sites are marked as red. (C) Motif organization of RCT domains from S. cerevisiae, S. pombe and H. Sapiens. Identity (black) and similarity (grey) of these motifs are indicated. (D) Overlay of the RCT domains from S. cerevisiae (red, PDB: 30WT), S. pombe (green, PDB: 2L3N) and $H$. Sapiens (yellow, PDB: $3 K 6 \mathrm{G}$ ). The conserved $\mathrm{RCT}_{M}$ and $\mathrm{RCT}_{\mathrm{C}}$ are circled. For clarification, $\mathrm{RCT}_{\mathrm{N}}$ in $S$. cerevisiae Rap1 is shown in transparent representation. (E) Comparison of $H_{s R C T}$ (yellow, PDB: 3K6G) with UBA domain from UBE2K (red, PDB: 3K9P) shows similar folds with different substrate-binding pockets. UBA of UBE2K use a pocket to bind ubiquitin (grey), opposite to RAP1 pocket used for TRF2-binding (blue). (F) Structural comparison of Rap ${ }_{B R C T}$ from S. cerevisiae (yellow, PDB: 2L42) with HsBRCA1 $1_{B R C 1}$ domain (red, PDB: 1LOB). 
Figure 3B) (Chen et al., 2008). Notably, the subtle difference in the linear motif determines its binding-preference for TRF1 or TRF2. TRF $1_{\text {TRFH }}$ recognizes TBM with a positively charged tail and a hydrophobic residue (F or I) at -2 position, while $\mathrm{TRF} 2_{\mathrm{TRFH}}$ prefers TBM with a polar residue $(\mathrm{Y}$ or $\mathrm{H})$ at -2 position. The binding specificities toward different proteins coincide exactly with the corresponding major function of TRF1 and TRF2 in telomere length regulation and telomere protection, respectively. Notably, not all proteins binding to a $\mathrm{TRF} 1_{\mathrm{TRFH}}$ or $\mathrm{TRF} 2_{\mathrm{TRFH}}$ domain are mediated by this conserved motif. For example, FBX4, an F-box protein in ubiquitin E3 ligase SCF, binds $\mathrm{TRF} 1_{\mathrm{TRFH}}$ using a different surface that partially overlaps with a TBM-binding pocket (Zeng et al., 2010). TIN2 $2_{\text {TRFH }}$ and Poz1 $1_{\text {TRFH }}$ also utilize TRFH domains to interact with their binding partners on similar surfaces (Figure 3A). Overall, the occurrence of TRFH domains in different telomeric proteins suggests the common evolutionary origin of these proteins. TRFH domains have gained significant structural and functional plasticity during evolution.

\section{RCT domain: a diversified protein-interaction domain}

The RCT domain is a protein-protein interaction module mediating RAP1 interaction with different partners. In mammalian and fission yeasts, this domain from RAP1 interacts with TRF2 and Taz1, respectively, targeting RAP1 to telomeres for telomere protection (Chen et al., 2011). Saccharomyces cerevisiae Rap1 uses its RCT domain to recruit Rif1/Rif2/Sir3/Sir4 in order to mediate telomere homeostasis and gene silencing (Moretti et al., 1994; Wotton and Shore, 1997; Moretti and Shore, 2001). RCT can be structurally divided into three motifs: $\mathrm{RCT}_{\mathrm{N}}, \mathrm{RCT}_{\mathrm{M}}$ and $\mathrm{RCT}_{\mathrm{C}}$ (Figure 3C). $\mathrm{RCT}_{\mathrm{M}}$ is the major protein-interaction motif with high structural conservation but low sequence identity in difference species (Figure $3 \mathrm{C}$ and D). It is a threehelix bundle that recognizes a helical peptide from binding partners, driven by hydrophobic interactions (Chen et al., 2011) (Figure 3D). Interestingly, this structural motif resembles an ubiquitin-associated (UBA) domain, but they bind protein partners by using opposite surfaces (Figure 3E). Whether RCT $_{M}$ of Rap1 binds ubiquitin needs further study. Meanwhile, ScRap1 and HsRap1 contain another threehelix motif $\left(\mathrm{RCT}_{\mathrm{C}}\right.$ ), which is absent in SpRap1 (Figure 3C). This motif is indicated by the significant sequence similarity between ScRap1 and HsRap1 (Li et al., 2000). The function of this $\mathrm{RCT}_{\mathrm{C}}$ motif remains to be addressed. In addition, ScRap1 has an exclusive N-terminal four-helix extension $\left(\mathrm{RCT}_{\mathrm{N}}\right)$, which is not found in human RAP1 and fission yeast Rap1. RCT $_{\mathrm{N}}$ of $S c$ Rap1 makes direct contact with $S c R i f 2$ AAA + domain (Shi et al., 2013) and is thus responsible for the specific functions of ScRap1. The structural divergence of the RCT domains may account for the diversified functions of Rap1 in different organisms.

\section{BRCT domain: a putative protein-interaction domain}

The BRCT domain is a common protein-interaction domain that usually recognizes phosphorylated peptides (Leung and Glover, 2011). BRCT domains are identified in the N-terminal regions of RAP1 from all species. The BRCT domain from ScRap1 can activate transcription and induce chromatin remodeling (Miyake et al., 2000), and the deletion of BRCT from HsRap1 diminishes the heterogeneity of human telomeres (Li and de Lange, 2003). However, the precise role of BRCT domain in telomere maintenance is unclear, and the interaction partners for BRCT domains remain unknown.

The canonical BRCT domain, as shown in BRCA1 BRCT domain, contains a central $\beta$-sheet sandwiched by two layers of helices: a bottom layer with three helices and a top layer with another two helices (Figure 3F). However, the NMR structure of $S c R a p 1_{\text {BRCT }}$ shows a loose-packed fold and has no top layer of helices (Figure 3F) (Zhang et al., 2011). This structural feature strongly suggests that Rap1 $1_{\mathrm{BRCT}}$ uses a protein-interaction interface that is different from the interfaces used by canonical BRCT domains. Although only one BRCT domain is present in the RAP1 molecule, a single shelterin complex contains two copies of RAP1 because of the dimerization of RAP1-interacting proteins TRF2 (or Taz1 in fission yeast). A small-angle X-ray scattering (SAXS) study of the intact TRF2-RAP1 complex reveals that two RAP $1_{\text {BRCT }}$ domains are closely packed (Gaullier et al., 2016). Thus, some proteins (especially phosphorylated proteins) might bind to the structural tandem BRCT domains in the context of the intact shelterin complex. It is expected that proteins interacting with BRCT domains of RAP1 and their functions in telomere regulation will be reported in the near future.

\section{Assembly of the shelterin complex}

Structural and biochemical studies suggest that shelterin complexes adopt a conserved assembly mechanism. The stable association of mammalian shelterin complex is mediated by five direct protein-protein interactions, 
namely, TRF1-TIN2, TRF2-TIN2, TRF2-RAP1, TIN2-TPP1 and POT1-TPP1 (Figure 1B). Analogously, the assembly of fission yeast shelterin complex is mediated by the following direct interactions: Taz1-Rap1, Rap1-Poz1, Pot1-Tpz1, Tpz1-Poz1 and Tpz1-Ccq1 (Figure 1C). These bipartite interactions have been structurally characterized, except the Pot1-Tpz1 and Tpz1-Ccq1 interactions. A striking feature observed from these structures is that all these interactions are mediated by the domain-peptide interaction mode. The TRF1-TIN2 interaction is dominated by a TRF $1_{\text {TRFH }}$ domain and a short TRFH-binding motif of TIN2 (TIN2 $2_{\text {TBM }}$ ). TRF2 binds TIN2 through two sites: one site is between a $\mathrm{TRF}_{\mathrm{TRFH}}$ domain and a TRFH-binding motif of TIN2, and the other is between a TIN2 ${ }_{\text {TRFH }}$ domain and a TIN2-binding motif of TRF2 $\left(\mathrm{TRF} 2_{\text {TBM }}\right)$. TIN2 $2_{\text {TRFH }}$ domain also recognizes a C-terminal short motif of TPP1. RAP1 uses its RCT domain to interact with a helical peptide of TRF2. The C-terminal domain of POT1, including an OB fold and a holiday junction-like domain, binds a long fragment of TPP1. Similar domain-peptide interaction modes are also adopted by all shelterin subcomplexes in fission yeast.

The selection of such a domain-peptide interaction mechanism presents several advantages for shelterin complex assembly. First, this domain-peptide interaction mode can coordinate different domains without altering the functions of individual domains and results in great evolutional plasticity. It might be essential to the conserved roles of rapidly-evolved shelterin proteins in telomere maintenance. It also ensures that these bipartite interactions could occur independently of the intact shelterin complex, as shelterin components are not in equal stoichiometry (Takai et al., 2010). Second, this domainpeptide interaction mode enables a single domain to recognize multiple proteins with a conserved linear motif, thereby providing an economical way for the integration of communication networks between shelterin proteins and other telomere-associating proteins, such as DNArepair and DNA-damage signaling proteins. A remarkable example is the recruitment of numerous proteins with diversified functions by the TRFH domains of TRF1 and TRF2 through a conserved binding motif. This process coordinates complicated interaction networks that are essential for telomere protection.

Moreover, the domain-peptide interaction mode provides an efficient way for regulating complex assembly or disassembly for fast response to external stimuli. Given that domain-peptide interactions usually have relatively small contacting surfaces compared with domain-domain interactions, the substitution or modification of a single residue greatly changes the binding affinity between proteins. One example is the disruption of TRF2-NBS1 interaction by $\mathrm{NBS1}^{\mathrm{S} 432}$ phosphorylation (Rai et al., 2017). Similarly, TERB1 ${ }^{\text {T648 }}$ phosphorylation abolishes TRF1TERB1 interaction (Pendlebury et al., 2017). NBS1 ${ }^{\mathrm{S} 432}$ and TERB1 $^{\mathrm{T}} 648$ are both located in TRFH-binding motifs (Figure 3B). In fact, the sequence alignment of TBMs from different proteins shows that most TBMs contain potential phosphorylation sites in the cores or vicinities of TBM sequences (Figure 3B). TBM sits in a shallow hydrophobic pocket surrounded by the acidic residues of $\mathrm{TRF}_{\mathrm{TRFH}}$ and TRF $2_{\text {TRFH }}$ (Figure 3B). Therefore, the negatively charged phosphate group deposited on a TBM cannot be accommodated into this TRFH pocket, and thus the interaction between TBM and TRFH is disrupted. In shelterin complexes, cell-cycle-dependent and DNA-damage-repsonsedependent phosphorylation or other modifications are expected to modulate complex assembly or disassembly in a highly organized way. It provides a feasible way for the regulation of shelterin functions in different cellular contexts.

This domain-peptide interaction mode enables us to design inhibitory peptides or small molecules that can efficiently compete for the targeted domain-peptide interactions in shelterin complexes. Examples of such peptides are cyclic peptides mimicking Apollo $_{\text {твм}}$, which were designed to target TRFH-binding pockets on TRF2 (Di Maro et al., 2014; Chen et al., 2018). These inhibitors can bind to $\mathrm{TRF} 2_{\mathrm{TRFH}}$ with high affinity and elicit DNA damage response in cancer cells (Di Maro et al., 2014). A triazolestapled peptide was also designed to inhibit the RAP1 $1_{\mathrm{RCT}^{-}}$ $\mathrm{TRF}_{\mathrm{RBM}}$ interaction (Ran et al., 2016). Other interactions in shelterin complexes are interesting targets for future rational design. These inhibitors not only serve as important research tools for determining the functional significance of a specific interaction in vivo but also useful in exploring therapeutic drugs for cancer treatment.

\section{Perspective}

To date, almost all the high-resolution structures of isolated domains and the shelterin subcomplexes have been reported. These structures have provided valuable information on shelterin recruitment, assembly and regulation. However, these data only provides an incomplete view on the whole shelterin complex given that an intact shelterin complex is not a simple adduct of these subcomplexes. As shown in the S. pombe Poz1-Tpz1-Rap1 complex, Tpz1 binding to Poz1 induces a conformational change in Poz1, allosterically enhancing the binding affinity of Poz1 with Rap1 (Kim et al., 2017). The same 
cooperative interaction occurs in human TIN2-TPP1-TRF2 complexes (Hu et al., 2017; Kim et al., 2017). Thus, the structure of an intact shelterin must be investigated so that the mechanisms of how shelterin components cooperate for telomere maintenance can be determined. Moreover, whether hundreds of shelterin complexes on the long tract of telomere repeats form any high-order structure remains to be addressed. A preliminary EM study of POT1-TPP1 in complex with 144-mer ssDNA indicates that 12 POT1-TPP1 heterodimer on ssDNA can form a compact and ordered structure, suggesting that the heterodimer exerts self-organization ability (Taylor et al., 2011). Therefore, the next challenge is the elucidation of the in vivo structural features of shelterin complexes in the long tracts of telomeric repeats. These features may provide insights into the mechanisms by which these high-order shelterin assemblies participate in telomere maintenance and protection.

Acknowledgments: This work was supported by grants from the National Natural Science Foundation of China (31470737 and 31670748). I apologize to all those whose work I have been unable to include due to space limitations.

Conflict of interest statement: The author declares no competing interests.

\section{References}

Azad, G.K. and Tomar, R.S. (2016). The multifunctional transcription factor Rap1: a regulator of yeast physiology. Front Biosci. 21, 918-930.

Baumann, P. and Cech, T.R. (2001). Pot1, the putative telomere end-binding protein in fission yeast and humans. Science 292, 1171-1175.

Benarroch-Popivker, D., Pisano, S., Mendez-Bermudez, A., Lototska, L., Kaur, P., Bauwens, S., Djerbi, N., Latrick, C.M., Fraisier, V., Pei, B., et al. (2016). TRF2-mediated control of telomere DNA topology as a mechanism for chromosome-end protection. Mol. Cell 61, 274-286.

Bianchi, A., Stansel, R.M., Fairall, L., Griffith, J.D., Rhodes, D., and de Lange, T. (1999). TRF1 binds a bipartite telomeric site with extreme spatial flexibility. EMBO J. 18, 5735-5744.

Broccoli, D., Smogorzewska, A., Chong, L., and de Lange, T. (1997). Human telomeres contain two distinct Myb-related proteins, TRF1 and TRF2. Nat. Genet. 17, 231-235.

Chen, Y., Yang, Y., van Overbeek, M., Donigian, J.R., Baciu, P., de Lange, T., and Lei, M. (2008). A shared docking motif in TRF1 and TRF2 used for differential recruitment of telomeric proteins. Science 319, 1092-1096.

Chen, Y., Rai, R., Zhou, Z.R., Kanoh, J., Ribeyre, C., Yang, Y., Zheng, H., Damay, P., Wang, F., Tsujii, H., et al. (2011). A conserved motif within RAP1 has diversified roles in telomere protection and regulation in different organisms. Nat. Struct. Mol. Biol. 18, 213-221.

Chen, L.Y., Redon, S., and Lingner, J. (2012). The human CST complex is a terminator of telomerase activity. Nature 488, 540-544.

Chen, C., Gu, P., Wu, J., Chen, X., Niu, S., Sun, H., Wu, L., Li, N., Peng, J., Shi, S., et al. (2017). Structural insights into POT1-TPP1 interaction and POT1 C-terminal mutations in human cancer. Nat. Commun. 8, 14929.

Chen, X., Liu, L., Chen, Y., Yang, Y., Yang, C.Y., Guo, T., Lei, M., Sun, H., and Wang, S. (2018). Cyclic peptidic mimetics of Apollo peptides targeting telomeric repeat binding factor 2 (TRF2) and Apollo interaction. ACS Med. Chem. Lett. 9, 507-511.

Churikov, D., Corda, Y., Luciano, P., and Geli, V. (2013). Cdc13 at a crossroads of telomerase action. Front. Oncol. 3, 39.

Conrad, M.N., Wright, J.H., Wolf, A.J., and Zakian, V.A. (1990). RAP1 protein interacts with yeast telomeres in vivo: overproduction alters telomere structure and decreases chromosome stability. Cell 63, 739-750.

Cooper, J.P., Nimmo, E.R., Allshire, R.C., and Cech, T.R. (1997). Regulation of telomere length and function by a Myb-domain protein in fission yeast. Nature $385,744-747$.

Cooper, J.P., Watanabe, Y., and Nurse, P. (1998). Fission yeast Taz1 protein is required for meiotic telomere clustering and recombination. Nature 392, 828-831.

Court, R., Chapman, L., Fairall, L., and Rhodes, D. (2005). How the human telomeric proteins TRF1 and TRF2 recognize telomeric DNA: a view from high-resolution crystal structures. EMBO Rep. 6, 39-45.

Croy, J.E., Altschuler, S.E., Grimm, N.E., and Wuttke, D.S. (2009). Nonadditivity in the recognition of single-stranded DNA by the Schizosaccharomyces pombe protection of telomeres 1 DNAbinding domain, Pot1-DBD. Biochemistry 48, 6864-6875.

de Lange, T. (2005). Shelterin: the protein complex that shapes and safeguards human telomeres. Genes Dev. 19, 2100-2110.

de Lange, T. (2009). How telomeres solve the end-protection problem. Science 326, 948-952.

Denchi, E.L. and de Lange, T. (2007). Protection of telomeres through independent control of ATM and ATR by TRF2 and POT1. Nature 448, 1068-1071.

Deng, W., Wu, J., Wang, F., Kanoh, J., Dehe, P.M., Inoue, H., Chen, J., and Lei, M. (2015). Fission yeast telomere-binding protein Taz1 is a functional but not a structural counterpart of human TRF1 and TRF2. Cell Res. 25, 881-884.

Di Maro, S., Zizza, P., Salvati, E., De Luca, V., Capasso, C., Fotticchia, I., Pagano, B., Marinelli, L., Gilson, E., Novellino, E., et al. (2014). Shading the TRF2 recruiting function: a new horizon in drug development. J. Am. Chem. Soc. 136, 16708-16711.

Dickey, T.H., McKercher, M.A., and Wuttke, D.S. (2013). Nonspecific recognition is achieved in Pot1pC through the use of multiple binding modes. Structure 21, 121-132.

Fairall, L., Chapman, L., Moss, H., de Lange, T., and Rhodes, D. (2001). Structure of the TRFH dimerization domain of the human telomeric proteins TRF1 and TRF2. Mol. Cell 8, 351-361.

Frescas, D. and de Lange, T. (2014). TRF2-tethered TIN2 can mediate telomere protection by TPP1/POT1. Mol. Cell Biol. 34, 1349-1362.

Gaullier, G., Miron, S., Pisano, S., Buisson, R., Le Bihan, Y.V., TellierLebegue, C., Messaoud, W., Roblin, P., Guimaraes, B.G., Thai, R., et al. (2016). A higher-order entity formed by the flexible assembly of RAP1 with TRF2. Nucleic Acids Res. 44, 1962-1976. 
Gelinas, A.D., Paschini, M., Reyes, F.E., Heroux, A., Batey, R.T., Lundblad, V., and Wuttke, D.S. (2009). Telomere capping proteins are structurally related to RPA with an additional telomere-specific domain. Proc. Natl. Acad. Sci. USA 106, 19298-19303.

Hanaoka, S., Nagadoi, A., Yoshimura, S., Aimoto, S., Li, B., de Lange, T., and Nishimura, Y. (2001). NMR structure of the hRap1 Myb motif reveals a canonical three-helix bundle lacking the positive surface charge typical of Myb DNA-binding domains. J. Mol. Biol. 312, 167-175.

Hockemeyer, D., Daniels, J.P., Takai, H., and de Lange, T. (2006). Recent expansion of the telomeric complex in rodents: two distinct POT1 proteins protect mouse telomeres. Cell 126, 63-77.

Hu, C., Rai, R., Huang, C., Broton, C., Long, J., Xu, Y., Xue, J., Lei, M., Chang, S., and Chen, Y. (2017). Structural and functional analyses of the mammalian TIN2-TPP1-TRF2 telomeric complex. Cell Res. 27, 1485-1502.

Kabir, S., Sfeir, A., and de Lange, T. (2010). Taking apart Rap1 An adaptor protein with telomeric and non-telomeric functions. Cell Cycle 9, 4061-4067.

Kanoh, J. and Ishikawa, F. (2001). spRap1 and spRif1, recruited to telomeres by Taz1, are essential for telomere function in fission yeast. Curr. Biol. 11, 1624-1630.

Kim, S.H., Kaminker, P., and Campisi, J. (1999). TIN2, a new regulator of telomere length in human cells. Nat. Genet. 23, 405-412.

Kim, J.K., Liu, J., Hu, X., Yu, C., Roskamp, K., Sankaran, B., Huang, L., Komives, E.A., and Qiao, F. (2017). Structural basis for shelterin bridge assembly. Mol. Cell 68, 698-714 e695.

Lei, M., Podell, E.R., Baumann, P., and Cech, T.R. (2003). DNA selfrecognition in the structure of Pot1 bound to telomeric singlestranded DNA. Nature 426, 198-203.

Lei, M., Podell, E.R., and Cech, T.R. (2004). Structure of human POT1 bound to telomeric single-stranded DNA provides a model for chromosome end-protection. Nat. Struct. Mol. Biol. 11, 1223-1229.

Leung, C.C. and Glover, J.N. (2011). BRCT domains: easy as one, two, three. Cell Cycle 10, 2461-2470.

Lewis, K.A. and Wuttke, D.S. (2012). Telomerase and telomereassociated proteins: structural insights into mechanism and evolution. Structure 20, 28-39.

Li, B. and de Lange, T. (2003). Rap1 affects the length and heterogeneity of human telomeres. Mol. Biol. Cell 14, 5060-5068.

Li, B., Oestreich, S., and de Lange, T. (2000). Identification of human Rap1: implications for telomere evolution. Cell 101, 471-483.

Martinez, P. and Blasco, M.A. (2015). Replicating through telomeres: a means to an end. Trends Biochem. Sci. 40, 504-515.

Martinez, P., Thanasoula, M., Carlos, A.R., Gomez-Lopez, G., Tejera, A.M., Schoeftner, S., Dominguez, O., Pisano, D.G., Tarsounas, M., and Blasco, M.A. (2010). Mammalian Rap1 controls telomere function and gene expression through binding to telomeric and extratelomeric sites. Nat. Cell Biol. 12, 768-780.

Miller, K.M. and Cooper, J.P. (2003). The telomere protein Taz1 is required to prevent and repair genomic DNA breaks. Mol. Cell 11, 303-313.

Miller, K.M., Ferreira, M.G., and Cooper, J.P. (2005). Taz1, Rap1 and Rif1 act both interdependently and independently to maintain telomeres. EMBO J. 24, 3128-3135.

Miller, K.M., Rog, O., and Cooper, J.P. (2006). Semi-conservative DNA replication through telomeres requires Taz1. Nature 440, 824-828.
Miyake, T., Hu, Y.F., Yu, D.S., and Li, R. (2000). A functional comparison of BRCA1 C-terminal domains in transcription activation and chromatin remodeling. J. Biol. Chem. 275, 40169-40173.

Miyake, Y., Nakamura, M., Nabetani, A., Shimamura, S., Tamura, M., Yonehara, S., Saito, M., and Ishikawa, F. (2009). RPA-like mammalian Ctc1-Stn1-Ten1 complex binds to single-stranded DNA and protects telomeres independently of the Pot1 pathway. Mol. Cell 36, 193-206.

Miyoshi, T., Kanoh, J., Saito, M., and Ishikawa, F. (2008). Fission yeast Pot1-Tpp1 protects telomeres and regulates telomere length. Science 320, 1341-1344.

Moretti, P. and Shore, D. (2001). Multiple interactions in Sir protein recruitment by Rap1p at silencers and telomeres in yeast. Mol. Cell Biol. 21, 8082-8094.

Moretti, P., Freeman, K., Coodly, L., and Shore, D. (1994). Evidence that a complex of SIR proteins interacts with the silencer and telomere-binding protein RAP1. Genes Dev. 8, 2257-2269.

Moser, B.A. and Nakamura, T.M. (2009). Protection and replication of telomeres in fission yeast. Biochem. Cell Biol. 87, 747-758.

Nandakumar, J. and Cech, T.R. (2013). Finding the end: recruitment of telomerase to telomeres. Nat. Rev. Mol. Cell Biol. 14, 69-82.

Nandakumar, J., Podell, E.R., and Cech, T.R. (2010). How telomeric protein POT1 avoids RNA to achieve specificity for singlestranded DNA. Proc. Natl. Acad. Sci. USA 107, 651-656.

Nugent, C.I., Hughes, T.R., Lue, N.F., and Lundblad, V. (1996). Cdc13p: a single-strand telomeric DNA-binding protein with a dual role in yeast telomere maintenance. Science 274 , 249-252.

O’Connor, M.S., Safari, A., Xin, H., Liu, D., and Songyang, Z. (2006). A critical role for TPP1 and TIN2 interaction in high-order telomeric complex assembly. Proc. Natl. Acad. Sci. USA 103, 11874-11879.

Palm, W. and de Lange, T. (2008). How shelterin protects mammalian telomeres. Annu. Rev. Genet. 42, 301-334.

Pendlebury, D.F., Fujiwara, Y., Tesmer, V.M., Smith, E.M., Shibuya, H., Watanabe, Y., and Nandakumar, J. (2017). Dissecting the telomere-inner nuclear membrane interface formed in meiosis. Nat. Struct. Mol. Biol. 24, 1064-1072.

Rai, R., Chen, Y., Lei, M., and Chang, S. (2016). TRF2-RAP1 is required to protect telomeres from engaging in homologous recombination-mediated deletions and fusions. Nat. Commun. 7, 10881.

Rai, R., Hu, C., Broton, C., Chen, Y., Lei, M., and Chang, S. (2017). NBS1 phosphorylation status dictates repair choice of dysfunctional telomeres. Mol. Cell 65, 801-817 e804.

Ran, X., Liu, L., Yang, C.Y., Lu, J., Chen, Y., Lei, M., and Wang, S. (2016). Design of high-affinity stapled peptides to target the repressor activator protein 1 (RAP1)/telomeric repeat-binding factor 2 (TRF2) protein-protein interaction in the shelterin complex. J. Med. Chem. 59, 328-334.

Rice, C. and Skordalakes, E. (2016). Structure and function of the telomeric CST complex. Comput. Struct. Biotechnol. J. 14, 161-167.

Rice, C., Shastrula, P.K., Kossenkov, A.V., Hills, R., Baird, D.M., Showe, L.C., Doukov, T., Janicki, S., and Skordalakes, E. (2017). Structural and functional analysis of the human POT1-TPP1 telomeric complex. Nat. Commun. 8, 14928.

Sfeir, A., Kosiyatrakul, S.T., Hockemeyer, D., MacRae, S.L., Karlseder, J., Schildkraut, C.L., and de Lange, T. (2009). Mammalian telomeres resemble fragile sites and require TRF1 for efficient replication. Cell 138, 90-103. 
Sfeir, A., Kabir, S., van Overbeek, M., Celli, G.B., and de Lange, T. (2010). Loss of Rap1 induces telomere recombination in the absence of NHEJ or a DNA damage signal. Science 327, 1657-1661.

Shi, T., Bunker, R.D., Mattarocci, S., Ribeyre, C., Faty, M., Gut, H., Scrima, A., Rass, U., Rubin, S.M., Shore, D., et al. (2013). Rif1 and Rif2 shape telomere function and architecture through multivalent Rap1 interactions. Cell 153, 1340-1353.

Smogorzewska, A. and de Lange, T. (2004). Regulation of telomerase by telomeric proteins. Annu. Rev. Biochem. 73, 177-208.

Sun, J., Yu, E.Y., Yang, Y., Confer, L.A., Sun, S.H., Wan, K., Lue, N.F., and Lei, M. (2009). Stn1-Ten1 is an Rpa2-Rpa3-like complex at telomeres. Genes. Dev. 23, 2900-2914.

Takai, K.K., Hooper, S., Blackwood, S., Gandhi, R., and de Lange, T. (2010). In vivo stoichiometry of shelterin components. J. Biol. Chem. 285, 1457-1467.

Takai, K.K., Kibe, T., Donigian, J.R., Frescas, D., and de Lange, T. (2011). Telomere protection by TPP1/POT1 requires tethering to TIN2. Mol. Cell 44, 647-659.

Taylor, D.J., Podell, E.R., Taatjes, D.J., and Cech, T.R. (2011). Multiple POT1-TPP1 proteins coat and compact long telomeric singlestranded DNA. J. Mol. Biol. 410, 10-17.

Tomita, K. and Cooper, J.P. (2008). Fission yeast Ccq1 is telomerase recruiter and local checkpoint controller. Genes Dev. 22, 3461-3474.

van Steensel, B. and de Lange, T. (1997). Control of telomere length by the human telomeric protein TRF1. Nature 385, 740-743.

Wang, F., Podell, E.R., Zaug, A.J., Yang, Y., Baciu, P., Cech, T.R., and Lei, M. (2007). The POT1-TPP1 telomere complex is a telomerase processivity factor. Nature $445,506-510$.
Wotton, D. and Shore, D. (1997). A novel Rap1p-interacting factor, Rif2p, cooperates with Rif1p to regulate telomere length in Saccharomyces cerevisiae. Genes Dev. 11, 748-760.

Wu, L., Multani, A.S., He, H., Cosme-Blanco, W., Deng, Y., Deng, J.M., Bachilo, O., Pathak, S., Tahara, H., Bailey, S.M., et al. (2006). Pot1 deficiency initiates DNA damage checkpoint activation and aberrant homologous recombination at telomeres. Cell 126, 49-62.

Wu, P., Takai, H., and de Lange, T. (2012). Telomeric 3' overhangs derive from resection by Exo1 and Apollo and fill-in by POT1bassociated CST. Cell 150, 39-52.

Xin, H., Liu, D., Wan, M., Safari, A., Kim, H., Sun, W., O'Connor, M.S., and Songyang, Z. (2007). TPP1 is a homologue of ciliate TEBP- $\beta$ and interacts with POT1 to recruit telomerase. Nature 445 , 559-562.

Xue, J., Chen, H., Wu, J., Takeuchi, M., Inoue, H., Liu, Y., Sun, H., Chen, Y., Kanoh, J., and Lei, M. (2017). Structure of the fission yeast S. pombe telomeric Tpz1-Poz1-Rap1 complex. Cell Res. 27, 1503-1520.

Zeng, Z., Wang, W., Yang, Y., Chen, Y., Yang, X., Diehl, J.A., Liu, X., and Lei, M. (2010). Structural basis of selective ubiquitination of TRF1 by SCFFbx4. Dev. Cell 18, 214-225.

Zhang, W., Zhang, J., Zhang, X., Xu, C., and Tu, X. (2011). Solution structure of Rap1 BRCT domain from Saccharomyces cerevisiae reveals a novel fold. Biochem. Biophys. Res. Commun. 404, 1055-1059.

Zimmermann, M., Kibe, T., Kabir, S., and de Lange, T. (2014). TRF1 negotiates TTAGGG repeat-associated replication problems by recruiting the BLM helicase and the TPP1/POT1 repressor of ATR signaling. Genes Dev. 28, 2477-2491. 